\title{
Tensor vastus intermedius: a review of its discovery, morphology and clinical importance
}

\author{
T. Franchi(i) \\ The University of Sheffield Medical School, Sheffield, United Kingdom \\ [Received: 8 September 2020; Accepted: 8 October 2020; Early publication date: 12 October 2020]
}

Background: This review aims to summarise the relevant literature surrounding the tensor vastus intermedius, a newly discovered muscle, and to discuss its morphology and potential clinical importance. No such review currently exists in the literature.

Materials and methods: A comprehensive literature search regarding the tensor vastus intermedius was performed using PubMed and Google Scholar in July 2020.

Principles of Evidence-Based Anatomy and the Anatomical Quality Assessment Tool were employed to ensure a high-level review. All relevant papers were included and citation tracking was performed to uncover further publications.

Results: The tensor vastus intermedius is found in the anterior compartment of the thigh and has a similar morphology to the other vasti muscles. It has four main variations and a consistent neurovascular supply. The muscle has been implicated in a number of case reports and surgical procedures, which are described in detail in this paper.

Conclusions: In the 4 years since the tensor vastus intermedius was formally described, a significant amount of work has been done to help us understand its structure and function. Further efforts are needed to gain a full picture as to its clinical importance. As such, it is recommended that this muscle should be acknowledged in anatomical education. (Folia Morphol 2021; 80, 4: 792-798)

Key words: tensor vastus intermedius, quadriceps femoris muscle group, quinticeps, extensor apparatus of the knee, anatomical discovery

\section{INTRODUCTION}

The musculature of the human body has been a fascination of anatomists for many centuries. In book two of his groundbreaking work De Humani Corporis Fabrica Libri Septem, Andreas Vesalius intricately catalogued the structure and function of each muscle he observed during dissection [26]. Indeed, the 14 muscle-men engravings found in this book are still considered to be "arguably the most important of all illustrations in the history of medical science" [16]. Whilst anatomy's long history means that the discovery of novel gross anatomy is now extremely unlikely [17], the development of new instruments and techniques does allow for original perspectives on familiar structures [19].

The standard characterisation of the quadriceps femoris muscle found in leading anatomy textbooks describes the group as being formed by the rectus femoris, vastus lateralis, vastus intermedius and vastus medialis $[7,21]$. This definition is also reflected in the standard anatomical understanding of non-specialist anatomists and clinicians. However, research

Address for correspondence: T. Franchi, MSc, FHEA, MAcadMEd, The University of Sheffield Medical School, Beech Hill Road, Sheffield, S10 2RX, United Kingdom, tel: +447913415780, e-mail: tpffranchi1@sheffield.ac.uk

This article is available in open access under Creative Common Attribution-Non-Commercial-No Derivatives 4.0 International (CC BY-NC-ND 4.0) license, allowing to download articles and share them with others as long as they credit the authors and the publisher, but without permission to change them in any way or use them commercially. 


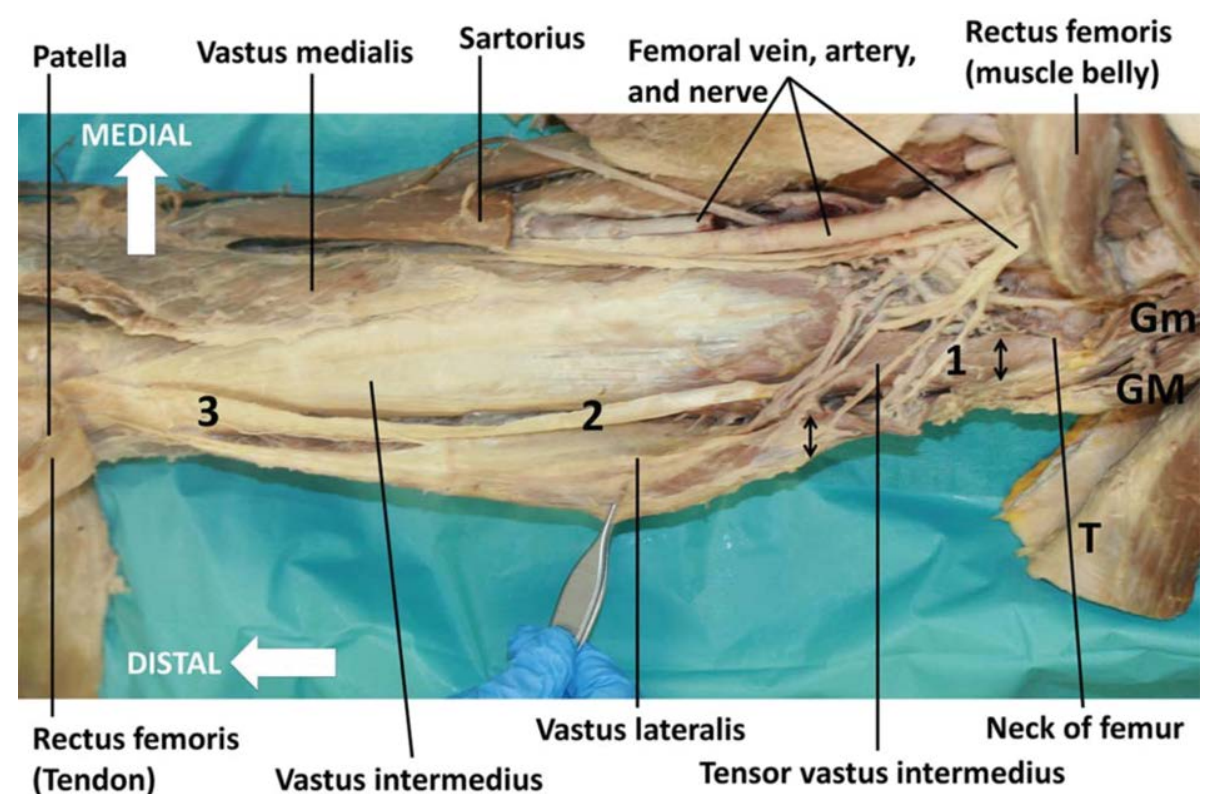

Figure 1. A dissection of tensor vastus intermedius. An anterior view of a left thigh, dissected to expose the quadriceps muscles, with rectus femoris, sartorius and tensor fasciae latae (T) transected and reflected. The muscle belly of tensor vastus intermedius (1), which can be seen between the muscle laminae of vastus lateralis and intermedius, merges into a broad aponeurosis (2) before becoming a tendinous structure (3). Gluteus medius (GM) and gluteus minimus $(\mathrm{Gm})$ are also shown. (From [9] — permission to reproduce was obtained from Author and Publisher [License Number: 4895961375084]).

carried out by a Swiss/Australian group in 2016 led to the identification, characterisation and naming of an additional muscle belly - the tensor vastus intermedius [9]. The anatomy of this newly discovered muscle, which is found between the vastus lateralis and vastus intermedius, is described in detail throughout this paper.

Previous authors investigating the quadriceps femoris muscle group had already reported the possibility of an additional muscle belly, but any conclusions that were made labelled this muscle as a variation, attributable to the vastus lateralis $[1,2,6,27,28]$. Two of these studies reported that an additional muscle belly was observed in 29\% [6] and 36\% [27] of dissected thighs, respectively. Interestingly, it was noted in a later study that the separation of the muscle bellies of the quadriceps femoris would align with both evolutionary and developmental biological trends [28].

The remainder of this paper will explore and discuss the morphology of the tensor vastus intermedius, as well as its potential importance in a number of clinical settings.

\section{MATERIALS AND METHODS}

A comprehensive literature search regarding the tensor vastus intermedius was performed using PubMed and Google Scholar in July 2020. Principles of
Evidence-Based Anatomy were employed when conducting this narrative review in order to ensure that the synopsis of primary anatomical research presented is of a high-level [15]. When designing inclusion and exclusion criteria it was decided to include all fulllength research articles, including case reports and university theses, as this is the first comprehensive review article on this topic and so it was considered appropriate to provide readers with a complete picture of the currently known research. Conference abstracts, letters to the editor and animal studies were excluded.

All studies deemed relevant for inclusion in this review were screened to assess any obvious risks of bias, by using the Anatomical Quality Assessment (AQUA) Tool [14]. No included studies were scored as high risk and therefore no studies were excluded. Citation tracking was performed to uncover further publications and, where relevant, data was extracted and is presented in the text of this paper. The papers' contents were then summarised in order to provide a critical analysis of the currently known information surrounding this muscle.

\section{RESULTS AND DISCUSSION}

\section{Morphology}

The tensor vastus intermedius is found in the anterior compartment of the thigh. It was first described 


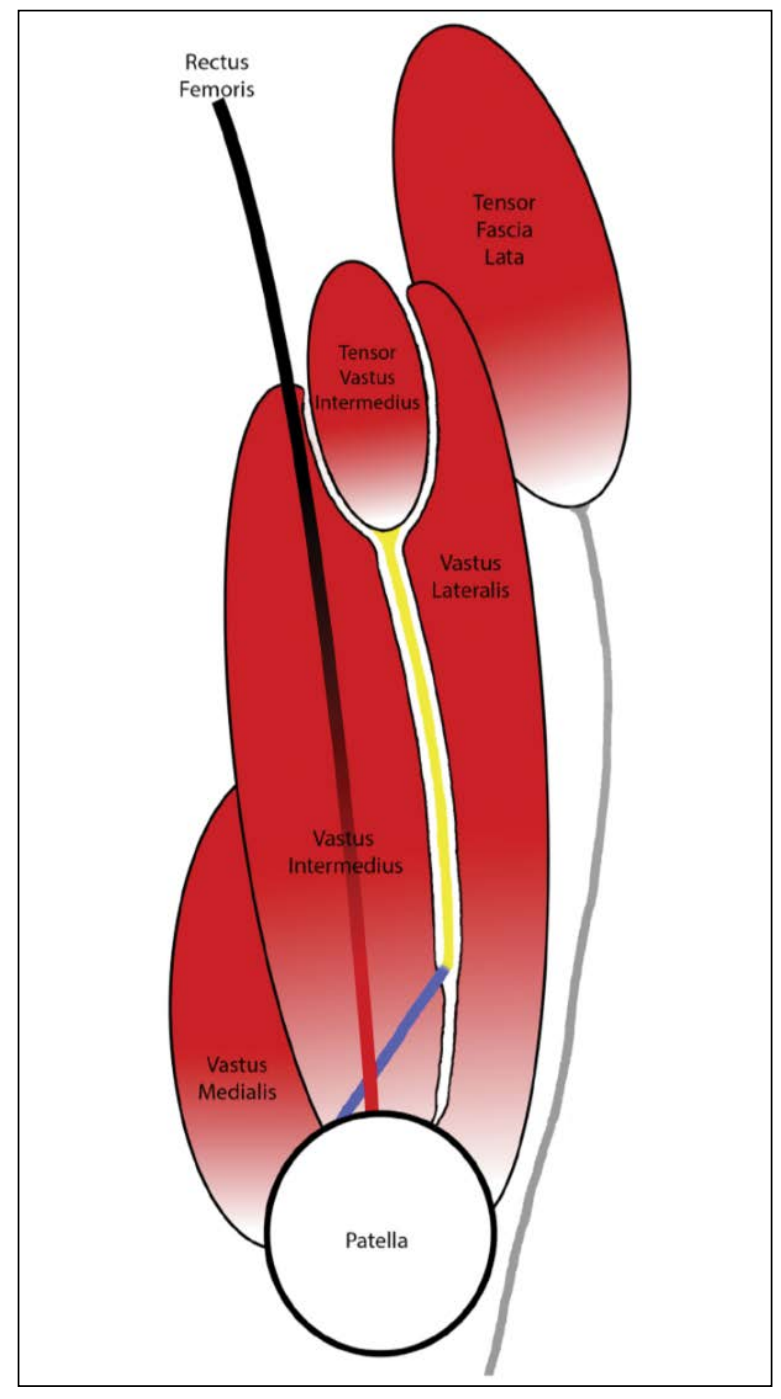

Figure 2. Schematic representation of tensor vastus intermedius. A schematic representation of the most common position for tensor vastus intermedius in relation to the quadriceps muscles and tensor fasciae latae. The aponeurosis of tensor vastus intermedius can be seen travelling between vastus lateralis and intermedius (yellow line), before becoming a tendon and turning obliquely (blue line). The iliotibial tract (grey line) is also shown. (From [22] permission to reproduce was obtained from Author and Publisher [License Number: 4895961023137]).

as a new muscle in a cadaver study involving 26 lower limbs, in which the researchers identified a clearly separate muscle belly between vastus lateralis and vastus intermedius in 22 of 26 cases [9]. In the remaining 4 cases, proximal separation of the muscle bellies was not possible, for reasons described later in this paper. The tensor vastus intermedius is covered anteriorly by rectus femoris [9], takes origin from the anteroinferior aspect of the greater trochanter of the femur distal to the intertrochantric line, and inserts into the medial aspect of the patellar base [9]. The position of tensor vastus intermedius in relation to its surrounding structures can be clearly seen in Figure 1.

Its morphology as a muscle follows a similar structure to the other vasti muscles, whereby it has a proximal muscle belly, which becomes a broad and flat aponeurosis before forming a tendinous structure which merges into the quadriceps femoris tendon [12]. The distal third of tensor vastus intermedius, the tendinous part, is interesting as it courses obliquely to its point of insertion [22]. This is shown in Figure 2. It has been hypothesised that this oblique nature of the tendon allows the muscle to counteract the forces produced by the medial muscles of the quadriceps femoris group [10].

Since its discovery, the anatomy of tensor vastus intermedius has been imaged and studied using both magnetic resonance imaging and sonography $[12,22]$. This has allowed a better understanding of its structure in relation to the surrounding muscles, revealing a multilayered lateral extensor apparatus of the knee. This can be clearly seen in Figure 3. Indeed, the morphology of the tensor vastus intermedius between the vastus lateralis and intermedius, as well as the iliotibial band, has been described as "onion-like muscle layers" whereby "individual layers come together and lie above each other" $[9,12]$. This beautiful architectural appearance has also been described as "layers of a husk of corn" [11] as the different overlying aponeurotic layers come together to form the quadriceps femoris tendon at the patella [13].

Whilst the most common form of tensor vastus intermedius is as described above, a number of studies have investigated variations in the muscle's morphology, which of course has direct implications into clinical practice. A study looking at 36 lower limbs reported the mean length of tensor vastus intermedius muscle bellies as $145.40 \mathrm{~mm}$ (standard deviation [SD] of $37.55 \mathrm{~mm}$ ) and length of aponeurosis as $193.55 \mathrm{~mm}$ (SD of $42.32 \mathrm{~mm}$ ), respectively [25]. Interestingly, this study also reported that both these measurements were greater in female cadavers compared to male ones.

Of even greater interest are the five distinct morphological variations described by two studies. Whilst the aponeurosis of tensor vastus intermedius is most commonly independent to vastus lateralis and intermedius, there appear to be frequent variations where it is either associated with vastus lateralis or intermedius, or both $[9,25]$. In these cases, it can be more difficult to identify the muscle in question, 


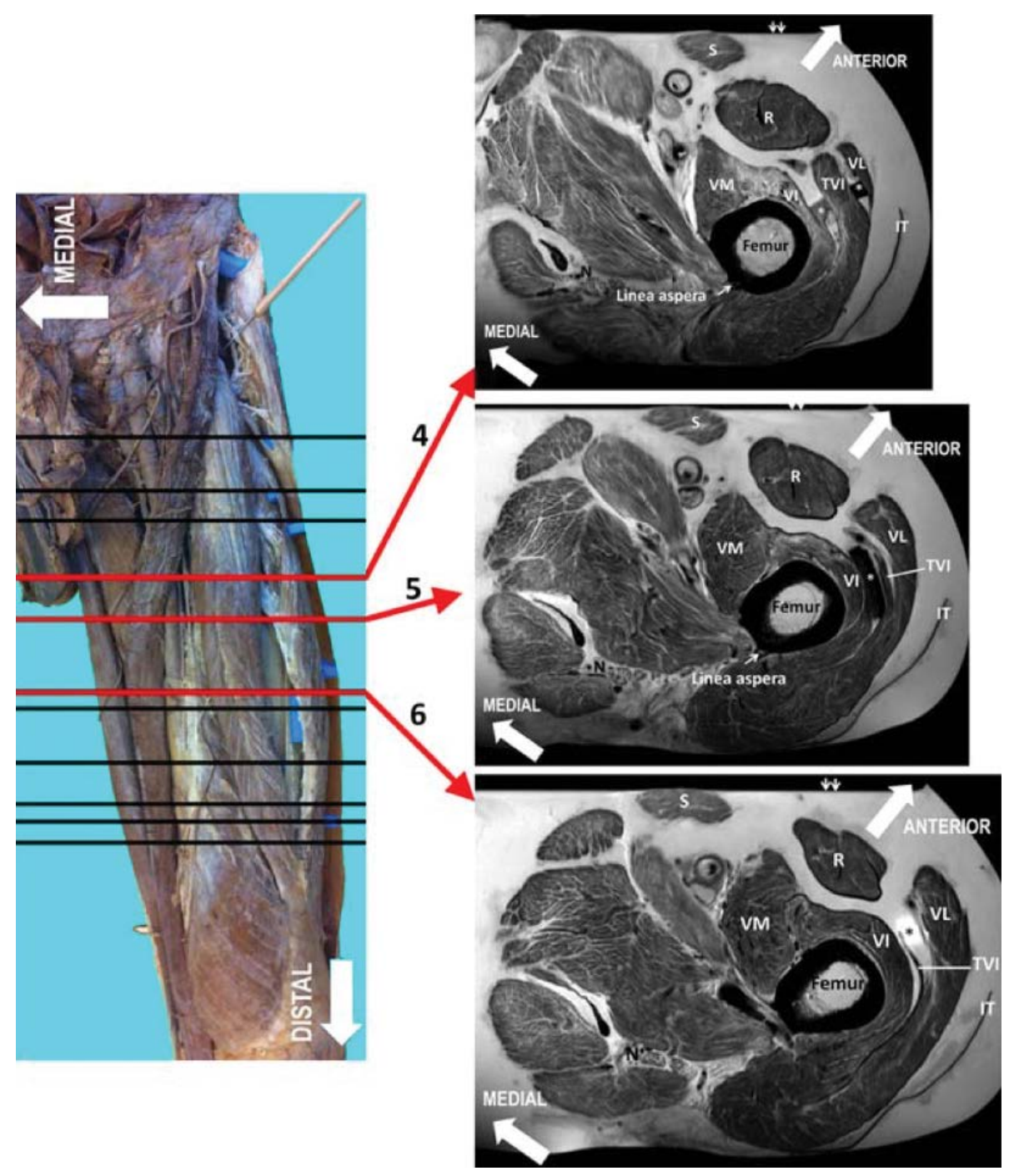

Figure 3. Magnetic resonance imaging (MRI) of tensor vastus intermedius. An anterior view of a left thigh alongside T2-weighted MRI. To allow better visualisation of muscles, foam blocks were placed between layers (marked in blue on the cadaver, and by an asterisk $\left({ }^{*}\right)$ on the MRI). Slice 4 (first MRI image) shows tensor vastus intermedius (TVI) muscle body between vastus lateralis (VL) and intermedius (VI). Slices 5 and 6 show tensor vastus intermedius thinning to an aponeurosis. Rectus femoris (R), sartorius (S), the iliotibial tract (IT) and the sciatic nerve (N) are also shown. (From [12] — permission to reproduce was obtained from Author and Publisher [License Number: 4895961225443]).

which perhaps is a factor involved in why it has only recently been described. There further appears to be a small number of cases where the tensor vastus intermedius is formed by two or more smaller intertwining muscle lamellae. The five reported variations in muscle morphology are outlined in Figure 4, and their relative prevalences are presented in Table 1. Interestingly however, a recent study investigating the tensor vastus intermedius found conflicting results when compared to the above. The study demonstrated the muscle in only seven of the 20 lower limbs studied [3], and the authors called into question the definition of this muscle. Perhaps, therefore, clarity is needed regarding the muscle's definition in order to allow for objective and comparable research to be undertaken.

A further crucial element to consider is that of the tensor vastus intermedius' blood and nerve supply, which was critical in establishing that this muscle is indeed an independent structure and not simply a part of the vastus lateralis. The detailed dissections undertaken in the main study referred to in this paper [9], demonstrated that tensor vastus intermedius receives separate vascular supply to vastus lateralis, through individual branches from the transverse branch of the lateral circumflex femoral artery, as well as side branches from its ascending branch [4]. Similarly with regards to innervation of the tensor vastus interme- 


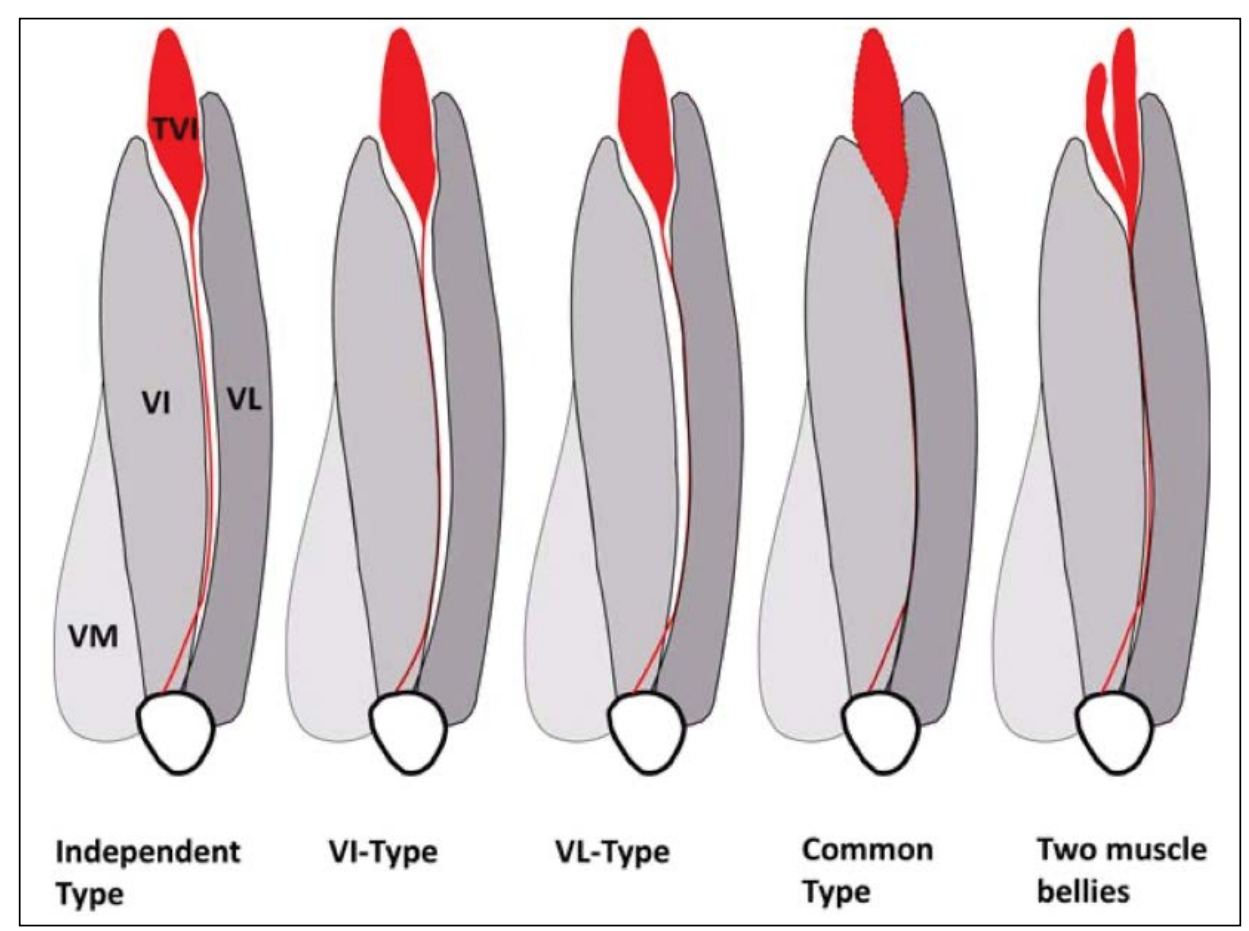

Figure 4. Variations of tensor vastus intermedius. A schematic representation of the variations in position of tensor vastus intermedius (TVI) in relation to vastus lateralis (VL) and intermedius (VI). A completely independent muscle belly and aponeurosis of tensor vastus intermedius represents the independent type. The case was labelled as VI-type if the aponeurosis was related to vastus intermedius, VL-type if related to vastus lateralis and common type if related to both muscles. In a small number of cadavers, regardless of type, there were multiple intervening muscle lamellae of the tensor vastus intermedius. These were labelled as two muscle bellies. (From [9] — permission to reproduce was obtained from Author and Publisher [License Number: 4895961375084]).

Table 1. Prevalence of tensor vastus intermedius variations

\begin{tabular}{lcccccc}
\hline Study & Number of LLs & Independent type & Vl-type & VL-type & Common type & Two muscle bellies \\
\hline A & 26 & $42.31 \%$ & $23.08 \%$ & $19.23 \%$ & $15.38 \%$ & $\ln 5$ cases \\
B & 36 & $33.33 \%$ & $8.33 \%$ & $30.56 \%$ & $27.78 \%$ & $\ln 3$ cases \\
Average & & $37.82 \%$ & $15.71 \%$ & $24.89 \%$ & $21.58 \%$ & $13.78 \%$
\end{tabular}

This table combines data from two studies (Study A: [9]; Study B: [25]) to provide some indication towards the prevalence of each variation of tensor vastus intermedius seen. Sixty-two lower limbs (LLS) were used between the two studies.

dius, the proximal muscle belly receives individual branches which originate from the lateral side of the posterior divisions of the femoral nerve [9, 25], with these branches being separable by dissection from the equivalent branches supplying the vastus lateralis and intermedius. Both these arteries and nerves are structures which are commonly studied by anatomy students, it would be worthwhile for teaching staff to encourage students to try and identify the muscle by tracing them on cadavers.

Based on the anatomy and morphology described above, it appears that tensor vastus intermedius has a significant role in controlling the motion of the patella, as well as in the extensor function of the knee [10]. Its aponeurosis is in close contact with the vastus intermedius and could potentially tighten it medially. In a similar fashion to the other muscles of the extensor compartment of the thigh, the tensor vastus intermedius is likely involved in both extension of the knee and prevention of flexion.

\section{Clinical importance}

Two case reports can be found in the literature that implicate the tensor vastus intermedius in the presenting pathology, and which align with the notion that the muscle is a distinct component of the extensor apparatus of the knee joint. The first [18], describes a 9-year-old girl with progressively limited knee flexion caused by an accessory quadriceps femoris muscle found between vastus lateralis and 
intermedius, which in retrospect, is clearly the tensor vastus intermedius. The second and more recent case [10], comes from the authors who first described the muscle, and documents a 62-year-old woman with an isolated ruptured tensor vastus intermedius tendon following a stumble and prevention of a fall. Despite there only being two case reports to date, the fact that this muscle is only newly described combined with the fact that its anatomical appearance and relationship with vastus lateralis and intermedius makes it hard to identify, likely means that many previous injuries have simply been attributed to the vastus lateralis.

The tensor vastus intermedius has also been identified in a recent cadaver report of a seven-headed quadriceps femoris. The authors note that an awareness of this additional muscle may provide clinicians with a further differential diagnosis when investigating anterior thigh pain [23]. This novel research may therefore allow clinicians to better characterise injuries of the quadriceps femoris muscle group. To date however, the exact role of the tensor vastus intermedius in the function of the quadriceps muscle group remains unclear [24].

An understanding of the tensor vastus intermedius also has implications on surgical applications and approaches. Anterolateral thigh flaps are a versatile and widely used flap used to provide skin, muscle and fascia for reconstructions following major head and neck surgery [20], breast mastectomy or coronary bypass surgery [5]. Variation in quadriceps femoris muscle anatomy affects surgeons' ability to harvest these flaps, and indeed authors have reported a case where aberrant fusion of the vastus lateralis and intermedius prevented successful harvest of an anterolateral thigh flap mid-surgery [20]. It is very likely that this fusion represented a common type variation of tensor vastus intermedius (as previously described with reference to Fig. 4). A further example of surgical importance is seen when considering the direct anterior approach to the hip, which is commonly used in performing total hip replacement surgery. Authors have demonstrated that if a distal extension of the procedure is needed, in the case of operative complications such as femoral fracture, there is an increased risk of damaging the neurovascular bundles associated with the quadriceps femoris [8]. This potentially damages the tensor vastus intermedius, and so having a complete anatomical understanding of the muscle and its neurovasculature can al- low surgeons to make operative decisions. Finally, as the tensor vastus intermedius inserts into the patella, a better understanding of the quadriceps femoris tendon would allow improved radiological and surgical interpretation of injuries to the extensor apparatus of the knee [29].

The above case reports and surgical examples demonstrate the significance of this novel research, and the importance that properly defining and understanding the tensor vastus intermedius has on a number of clinical applications. There is therefore also an argument to be made for including this muscle in the required content when designing and delivering anatomical education programmes to anatomy and medical students.

\section{CONCLUSIONS}

Whilst discovering novel gross anatomy is rare, better characterising and understanding the structures which anatomists see still furthers our knowledge and appreciation for the human body. In the 4 years since the tensor vastus intermedius was formally described [9], a significant amount of work has been done to help us understand its structure and function. However, the answer to the latter point, specifically in relation to its contribution to the quadriceps muscle group, is still largely unanswered.

This paper has extensively described the morphology of this muscle, touching on both its anatomical variations and independent neurovascular supply. Further, the cadaver studies documenting this muscle are necessary for a number of reasons: "to better understand the biomechanical limitations of this muscle; to define the implications on surgical approaches of the hip and thigh; and to discover how this muscle can be utilised in surgical procedures" [4]. Indeed, to answer these questions, a strong understanding of the muscle's anatomy is required. Upon reflection on the anatomy of this new muscle, it appears to be similar in morphology to the plantaris muscle. Perhaps therefore there are synergies to be made between their structures and functions.

Whilst the researchers in this field are starting to gain a clearer picture of the role tensor vastus intermedius has in both normal and pathological states, further studies, both in cadavers and living participants, are needed to fully uncover the story of this newly discovered muscle.

\section{Conflict of interest: None declared}




\section{REFERENCES}

1. Becker I, Woodley SJ, Baxter GD. Gross morphology of the vastus lateralis muscle: An anatomical review. Clin Anat. 2009; 22(4): 436-450, doi: 10.1002/ca.20792, indexed in Pubmed: 19306318.

2. Becker I, Baxter GD, Woodley SJ. The vastus lateralis muscle: an anatomical investigation. Clin Anat. 2010; 23(5): 575-585, doi: 10.1002/ca.20974, indexed in Pubmed: 20309954.

3. Bonnechère B, Louryan S, Feipel V. Triceps, quadriceps or pentaceps femoris? Need for proper muscle definition. Morphologie. 2020; 104(345): 77-84, doi: 10.1016/j. morpho.2019.06.001, indexed in Pubmed: 31296371.

4. Collins CT. Anatomical investigation of the tensor vastus intermedius in the quadriceps muscle group. MSc Thesis, University of North Texas 2017.

5. Goel S, Arora J, Mehta V, et al. Unusual disposition of lateral circumflex femoral artery: Anatomical description and clinical implications. World J Clin Cases. 2015; 3(1): 85-88, doi: 10.12998/wjcc.v3.11.85, indexed in Pubmed: 25610855.

6. Golland JA, Mahon M, Willan PL. Anatomical variations in human quadriceps femoris muscles. J Anat. 1986; 146(1): 263-264.

7. Gosling JA, Harris PF, Humpherson JR, Whitmore I, Willan PLT. Human anatomy: color atlas and textbook. Elsevier and Mosby, Missouri 2008.

8. Grob K, Monahan R, Gilbey H, et al. Distal extension of the direct anterior approach to the hip poses risk to neurovascular structures: an anatomical study. J Bone Joint Surg Am. 2015; 97(2): 126-132, doi: 10.2106/JBJS.N.00551, indexed in Pubmed: 25609439.

9. Grob K, Ackland T, Kuster MS, et al. A newly discovered muscle: The tensor of the vastus intermedius. Clin Anat. 2016; 29(2): 256-263, doi: 10.1002/ca.22680, indexed in Pubmed: 26732825.

10. Grob K, Fretz C, Kuster MS. Knee pain associated with rupture of tensor vastus intermedius, a newly discovered muscle: a case report. J Clin Case Rep. 2016; 6(7), doi: 10.4172/2165-7920.1000828.

11. Grob K, Manestar M, Filgueira L, et al. New insight in the architecture of the quadriceps tendon. J Exp Orthop. 2016; 3(1): 32, doi: 10.1186/s40634-016-0068-y, indexed in Pubmed: 27813020.

12. Grob K, Manestar M, Gascho D, et al. Magnetic resonance imaging of the tensor vastus intermedius: A topographic study based on anatomical dissections. Clin Anat. 2017; 30(8): 1096-1102, doi: 10.1002/ca.22981, indexed in Pubmed: 28833609.

13. Grob K, Manestar M, Filgueira L, et al. The interaction between the vastus medialis and vastus intermedius and its influence on the extensor apparatus of the knee joint. Knee Surg Sports Traumatol Arthrosc. 2018; 26(3): 727-738, doi: 10.1007/s00167-016-4396-3, indexed in Pubmed: 28124107.

14. Henry BM, Tomaszewski KA, Ramakrishnan PK, et al. Development of the anatomical quality assessment (AQUA) tool for the quality assessment of anatomical studies included in meta-analyses and systematic reviews. Clin
Anat. 2017; 30(1): 6-13, doi: 10.1002/ca.22799, indexed in Pubmed: 27718281.

15. Henry BM, Tomaszewski KA, Walocha JA. Methods of Evidence-Based Anatomy: a guide to conducting systematic reviews and meta-analysis of anatomical studies. Ann Anat. 2016; 205: 16-21, doi: 10.1016/j. aanat.2015.12.002, indexed in Pubmed: 26844627.

16. Kemp M. A drawing for the Fabrica; and some thoughts upon the Vesalius muscle-men. Med Hist. 1970; 14(3): 277-288, doi: 10.1017/s002572730001557x, indexed in Pubmed: 4921979.

17. Kumar A, Ghosh SK, Faiq MA, et al. A brief review of recent discoveries in human anatomy. QJM. 2019; 112(8): 567-573, doi: 10.1093/qjmed/hcy241, indexed in Pubmed: 30335170.

18. Labbé JL, Peres O, Leclair O, et al. Progressive limitation of knee flexion secondary to an accessory quinticeps femoris muscle in a child: a case report and literature review. J Bone Joint Surg Br. 2011; 93(11): 1568-1570, doi: 10.1302/0301-620X.93B11.27396, indexed in Pubmed: 22058313.

19. Neumann PE. Another new organ! is this a golden age of discovery in anatomy? Clin Anat. 2018; 31(5): 648-649, doi: 10.1002/ca.23184, indexed in Pubmed: 29664145.

20. Omakobia E, Liew C, Berridge N, et al. A rare case of aberrant quadriceps muscle anatomy preventing anterolateral thigh flap harvest. JPRAS Open. 2016; 7: 19-22, doi: 10.1016/j.jpra.2016.01.002.

21. Paulsen F, Waschke J. Sobotta atlas of anatomy. Elsevier and Urban \& Fischer, Munich 2018.

22. Rajasekaran S, Hall MM. Sonographic appearance of the tensor of the vastus intermedius. PM R. 2016; 8(10): 1020-1023, doi: 10.1016/j.pmrj.2016.04.002, indexed in Pubmed: 27108157.

23. Ruzik $K$, Waśniewska $A$, Olewnik $t$, et al. Unusual case report of seven-headed quadriceps femoris muscle. Surg Radiol Anat. 2020; 42(10): 1225-1229, doi: 10.1007/ s00276-020-02472-0, indexed in Pubmed: 32318799.

24. Sahinis C, Kellis E, Galanis N, et al. Intra- and inter-muscular differences in the cross-sectional area of the quadriceps muscles assessed by extended field-of-view ultrasonography. Med Ultrason. 2020; 22(2): 152-158, doi: 10.11152/ mu-2302, indexed in Pubmed: 32190847.

25. Veeramani R, Gnanasekaran D. Morphometric study of tensor of vastus intermedius in South Indian population. Anat Cell Biol. 2017; 50(1): 7-11, doi: 10.5115/ acb.2017.50.1.7, indexed in Pubmed: 28417049.

26. Vesalius A. De humani corporis fabrica libri septem. Johannes Oporinus, Basel 1543.

27. Willan PL, Mahon M, Golland JA. Morphological variations of the human vastus lateralis muscle. J Anat. 1990; 168: 235-239, indexed in Pubmed: 2323995.

28. Willan PLT, Ransome JA, Mahon M. Variability in human quadriceps muscles: quantitative study and review of clinical literature. Clin Anat. 2002; 15(2): 116-128, doi: 10.1002/ca.1106, indexed in Pubmed: 11877790.

29. Yablon CM, Pai D, Dong Q, et al. Magnetic resonance imaging of the extensor mechanism. Magn Reson Imaging Clin N Am. 2014; 22(4): 601-620, doi: 10.1016/j. mric.2014.07.004, indexed in Pubmed: 25442025. 